\title{
Two Distinct Human Medial Temporal Complexes
}

\author{
Ahmad Yousef ${ }^{1}$ \\ ${ }^{1}$ School of Computational Science and Engineering, McMaster University, Hamilton, Ontario, Canada \\ *Correspondence: mohamas2@mcmaster.ca
}

\begin{abstract}
We had previously discussed that hMT+ should have two distinct regions, one is connected to the central retina, and another is connected to the retinal peripheries; and both of these distinct regions generate different perceived speed. Namely, in the central region, the stimulus appears to move much slower, see references $1 \& 2$; in the peripheral region however, it appears to move faster and even with reversed direction, see reference 4 . This article, however, provides evidence that repeated symmetric patterns are perceived to move much slower than repeated asymmetric patterns (RAP); additionally, asymmetric but not symmetric patterns tends to generate illusory motion reversals.

Evidences which conclude that symmetric, but not asymmetric patterns are preferred to be processed by the central retina; an additional characteristic that adds further distinction between the central and the peripheral hMT+ regions.
\end{abstract}

conclusively speaking, we therefore may say, the central retina and its corresponding neurons in $\mathrm{hMT}+$ perceives the apparent motion to move slower, however, the peripheral retina and its corresponding neurons in hMT+ perceives the apparent motion not only moving faster but it may trigger illusory motion reversals, see reference 4 . The present study, however, shows how the geometry (repeated symmetric versus asymmetric patterns) affects the apparent speed. Findings that may evidence the hidden important roles of saccadic eye movement, pupil size, and lens shape variations, see reference 8; namely, how their different dynamics for different stimulus may feed either the central retina or the retinal peripheries. Eventually, we now have further clear distinctions between central and peripheral retina and their corresponding hMT+ regions.

Materials \& Methods Introduction

Sinha et al. had offered outstanding results when they showed the foveal

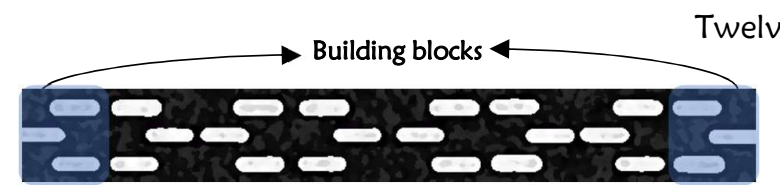

Twelve human subjects with dilated cones respond to light much slower Repeated Symmetric Pattern pupils were recruited to participate in this study. The experiment has two session, than cones located in the periphery, see reference 3 . They recorded signals directly from the cones, confirming that peripheral cones respond to light 30 milliseconds faster than foveal cones. Interestingly, these findings have serious implications in psychophysics, namely, the motion perceived in the central retina is way slower, see reference 2 . Historically speaking, the first scientist who discovered that the perception of the speed can be significantly reduced but by decreasing the contrast, was Peter Thompson, see reference 5 and 6. Further analysis shows that the reason behind Thompson's findings could be because of the pupil constriction induced by low contrast visual stimulation, see reference 7 . Constriction that may obliged the stimulus to be mostly processed by the central, but not the peripheral retina. Generally and the first session starts with asymmetric pattern for 30 seconds, then the symmetric pattern, and the session had been flipped for counterbalancing purposes, see reference 9, Both of the stimuli (symmetric and asymmetric) subtend 12.9 DVA length and 1.37 DVA width and consisted of 8 'building blocks', and both of the stimuli run at constant speed of 12.9 DVA/sec. Each human subject has to experience the experiment for three times for data finetuning. We intentionally built the stimuli with high speed to test whether illusory motion reversal will appear, and with which pattern.

Astonishingly, the symmetric pattern stimulus didn't show any illusory motion reversals, but the asymmetric stimulus shows a lot of motion reversals. Data Recording

We show all of the stimuli to each human subject in the beginning of the experiment and requested from 
them to consider the

repeated asymmetric

patterns stimulus to be the frame of reference. Namely, the frame of reference will be presented before the repeated symmetric patterns stimulus for one session, and after it in another session for counterbalancing purposes. The collected data are then analyzed using the statistical means, namely, the average and the standard error of the mean.

Results and Conclusion

As shown in the up-central infographic, there is significant speed declaration against the repeated symmetric patterns stimulus. Through sophisticated neural processes in several cortical and subcortical regions; the central retina, however, seems to trigger repulsive behavior possibly through systematic saccades against the repeated asymmetric but not symmetric patterns; saccades that may assist the peripheral retina to receive \& process its favorable patterns; and thus, greater speed of the apparent motion. Important to mention, observers had noticed when the perceived motion of the 'building blocks' is decelerated due the "symmetry", the size of the building blocks, compared to RAP, are perceived dilated (dilation mean value $\approx 0.26$, and $S E M \approx 0.043$ ). The present findings along with the previous findings show outstanding evidences that the central retina and its corresponding cortical neurons, especially those within hMT+ region had so different spatiotemporal properties than the peripheral retina and its hMT+ neurons. The aforementioned facts may give significant credibility to confidential say, there are two distinct hMT+ regions; classifying those regions with sharp mapping bounderies, however, will require professional visual tasks, fancy brain imaging machines, in addition to, high frame rates per second eye tracker.

Essential Notifications

Readers should have direct access to the stimuli, see reference 9. Advisably, download the videos for the best quality. Be sure that you have dilated pupils before the experiments, if you don't see illusory motion reversals in the experiment one's frame of reference, then you may not ready. In order to dilate the pupil, watch the video in fifty percent brightness, and have some caffeine intake, see reference 10 . During the experiment kindly follow the previously mentioned instructions in the 'materials \& method' section. We wish to collaborate with scholars who have access to several brain imaging machines, namely, MRI, EEC, and MEG, in addition to professional eye-tracker facilities in challenging investigations.

Scholars who wish to acquire high temporal resolution neurophysiological signals from different nodes in $\mathrm{hMT}+$ region to distinguish between the central and the peripheral hMT+ through the present experiments in order to draw sharp classifiers between these two distinct hMT+ regions are extremely welcome.

Enquiries may be sent to the author. Finally, we had to confirm here that repeated symmetric patterns stimulus had not produced any illusory motion reversals, confirming that these kinds of stimuli preferred to be processed by the central retina!

Transactional References

[1] Traschütz etal., 2012. Speed change detection in foveal and peripheral vision. Vision Research.

[2] Yousef, A. 2019. "Spatial Attention Dilates Time and Length." PsyArXiv. doi:10.31234/osf.io/qazj9.

[3] Sinha, etal. (2017). Cellular and circuit mechanisms shaping the perceptual properties of the primate fovea. Cell Journal.

[4] Yousef, A. 2019. "Retinal Peripheries Generate Illusory Motion Reversals." PsyArXiv. August, 2019.

doi:10.31234/osf.io/gfmpe.

[5] Thompson (1982). Perceived rate of movement depends on contrast. Vision Research.

[6] Stone \& Thompson (1992) Human speed perception is contrast dependent. Vision Research.

[7] Wang, et al., (2014). Modulation of stimulus contrast on the human pupil orienting response. EJN, 2014.

[8] Yousef, A. 2019. "Linking Dynamics Between Pupil and Lens." engrXiv. doi:10.31224/osf.io/9c3vk.

[9] You may find the stimuli in the following link: https://drive.google.com/drive/folders/lgQu90gFdMiOcgnyE40j YeoXLGB_C8tFw

[10] Abokyi, etal.,(2017). Caffeine intake is associated with pupil dilation and enhanced accommodation. Eye (Lond) Journal. 